\title{
Percutaneous Cortical Screw Fixation as a Method for Posterior Spinal Stabilization
}

\author{
Andrew S Jack, Wyatt L Ramey, Rod J Oskouian, Robert A Hart, Jeffrey S Roh \\ Department of Neurosurgery, Swedish Neuroscience Institute, Cherry Hill Swedish Medical Center, USA
}

Corresponding Author: Andrew S Jack, MD, PhD Department of Neurosurgery, Swedish Neuroscience Institute, Cherry Hill Swedish Medical Center, 550 17th Ave \#540, Seattle,
Washington, USA, WA 98122

Tel: +1-206-320-3782

Fax: +1-206-386-2700

E-mail: asjack@ualberta.ca

Received: March 25, 2019 Revised: May 27, 2019 Accepted: May 28, 2019
Objective: Novel minimally invasive surgery (MIS) approaches and instrumentation such as cortical screws (CS) have recently become commonplace for treating spinal disease. CS comparability to pedicle screws (PS) with respect to safety and mechanical stability, however, have yet to be determined. To our knowledge, this is the first report to describe a percutaneous CS fixation technique with subsequent open anatomical dissection and describe its feasibility compared to PS fixation. Methods: Fresh frozen cadavers were used for fluoroscopy-guided placement of a total of 20 percutaneous PS and CS. Standard percutaneous PS fixation was performed in cadavers from L1-L5 on one-side followed by CS being placed on the other side. Open anatomical dissection was then performed to confirm placement and compare pedicle breach incidence between PS and CS. Results: Both percutaneous PS and CS were easily placed without difficulty. Dissection revealed no breaches of either construct, and as such no statistical comparison was possible. At one-level however, a CS was seen breaching the posterior-third lateral vertebral body (VB) just under the superior end-plate. Two screw threads exposed were visualized well away from any entering and/or exiting foraminal or paraspinal neurovascular structures. Conclusion: Here, we report a novel study of percutaneous CS insertion with open cadaveric dissection comparing it to PS, incorporating the advantages of both MIS and CS fixation. Although feasible and technically comparable to PS in this report, further study comparing these two techniques and rigorous patient selection for its application are necessary.

Key Words: Cortical screw, Pedicle screw, Instrumentation, Fusion, Spine

\section{INTRODUCTION}

Pedicle screw (PS) spine fixation is considered to be the gold standard for achieving posterior spinal stabilization ${ }^{23,13,18)}$. However, PS placement is not without its drawbacks including, among others, extensive lateral dissection resulting in increased blood loss and postoperative pain ${ }^{3,13)}$. Since PS fixation was first described $^{18)}$, numerous other approaches and instruments have emerged for achieving spinal stabilization. One more recently described is the use of cortical screws $(\mathrm{CS})^{2,3,13,19)}$. As opposed to the more traditional lateral-to-medial approach for PS placement, CS consist of a medial-to-lateral and caudal-to-rostral approach for pedicle cannulation and screw placement. This trajectory, has been said to require less soft tissue dissection ${ }^{3)}$, and although shorter, has mainly cortical bone purchase resulting in biomechanical strength comparable to that of $\mathrm{PS}^{2,17)}$.

In addition to the advancement of spinal fixation techniques, a parallel trend toward more minimally invasive approaches has emerged over the past decade. Similarly to CS placement, percu- taneous PS placement has also been shown to require less soft tissue dissection, decreased blood loss, and postoperative pain compared to more open techniques ${ }^{8,9}$. Few studies to date have investigated the use of CS in comparison to traditional PS techniques, and as of yet, none have described a minimally invasive, percutaneous approach for CS-placement with subsequent open dissection for direct examination of its potential complications ${ }^{9,16)}$. In this paper, we aim to accurately describe this novel technique while also describing post-placement CS fixation through open anatomical cadaveric dissection in comparison to PS.

\section{METHODS AND TECHNIQUE}

Fresh frozen cadavers (mean age 80 years-old) were used for fluoroscopically-guided percutaneous placement of $20 \mathrm{CS}$ and PS. Cadavers were positioned prone and fluoroscopy used to mark the location of the initial entry point on the skin surface. A small skin incision (approximately $1.5 \mathrm{~cm}$ ) was made to allow placement of each CS. Five levels (L1-L5) were chosen to be instrumented on each cadaver. For each CS, a Jamshidi needle 
was used and tapped into place under fluoroscopy with a K-wire being placed through this into the more ventral vertebral body. The Jamshidi needle was then removed and a cannulated pedicle tap then passed over the K-wire using fluoroscopic guidance to ensure appropriate trajectory. This created a threaded trajectory for CS placement. Finally, the cannulated CS were inserted over the K-wire until in satisfactory position. The trajectory for CS placement was medial-to-to-lateral and caudal-to-rostral. Radiographic landmarks for the CS were as follows: entry point at the inferomedial aspect of the pedicle and final screw tip position superolateral pedicle on anteroposterior (AP) fluoroscopy (7 o'clock to 1 o'clock for the right pedicle and correspondingly 5 o'clock to 11 o'clock for the left pedicle if a CS were to be placed). On lateral fluoroscopy, the trajectory of each screw was caudal to rostral with the screw tip end being located slightly inferior to the superior vertebral body endplate. Screw sizes utilized were $6.5 \mathrm{~mm} \times 45 \mathrm{~mm}$ (Viper 2, Dupuy-Synthes, Raynham, MA) at all levels as shown in Figure 1. As there was no radiographic imaging prior to screw placement to accurately measure the pedicle size, the size of screw chosen was based on this being the most common size of screw used for the lumbar spine (in our experience), and the fluoroscopic appearance of the pedicle. Upon confirming satisfactory position of each cortical screw placed, a rod was tunneled through the " $x$-tabs" of the heads of the cortical screws (seen in Fig. 1) without diffi-

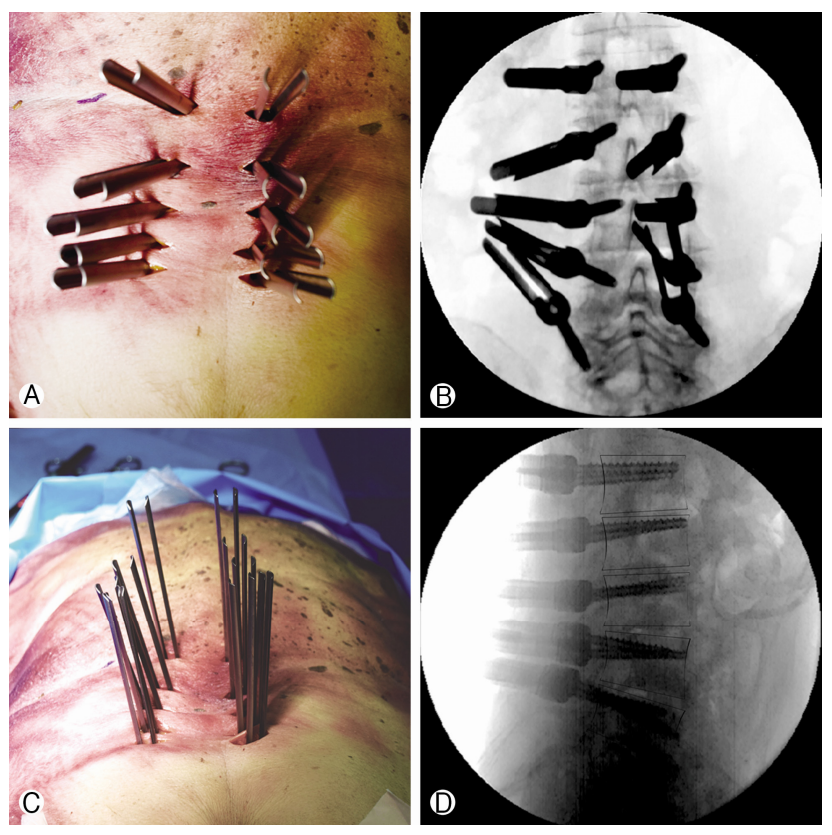

Fig. 1. Anteroposterior anatomical (panel (A)) and radiographic (panel (B)) depiction of the pedicle screw (PS, left-side of each respective panel) and cortical screw (CS, right-side of each respective panel) entry point and trajectory after screw insertion. Shown in panel (C) is the alignment of the screws (PS on the left-side and CS on the right-side of the panel) just prior to rod insertion and lateral radiographic depiction (panel (D)) of the trajectory of PS and CS. culty and secured in place using locking caps.

In order to compare percutaneous CS placement with standard percutaneous PS placement, each cadaver had unilateral CS placed (right-sided instrumentation, Fig. 1) and contralateral PS placed (left-sided instrumentation, Fig. 1). This helped in allowing each cadaver to serve as an internal anatomical control. Multiple small (approximately $1.5 \mathrm{~cm}$ ) incisions were made over the PS entry point, and the same series of steps were taken for PS placement as for CS placement. In comparison to CS, the trajectory for PS placement was lateral-to-medial and parallel to the superior endplate at each vertebral level. Radiographic landmarks for the PS were as follows: entry point at the lateral aspect of the pedicle and final screw tip position medial pedicle on AP fluoroscopy. On lateral fluoroscopy, the trajectory of each screw was parallel to the superior endplate with the screw tip end being located into the ventral third of the vertebral body. For comparison, PS sizes utilized were identical to CS. Upon confirming satisfactory position the PS, a rod was tunneled and secured in place using locking caps.

Anatomical confirmation was then performed by open dissection. A standard midline approach was taken with subperiosteal dissection of the lamina below and lateral reflection of the paraspinal soft tissue as shown in Figure 2. Entry points for each CS and PS were inspected (Fig. 2). Full laminectomies of $\mathrm{L} 1$ to $\mathrm{L} 5$ were performed, in addition to lateral vertebral dissection exposing the pedicles and vertebral bodies circumferentially (Fig. 2). The latter was performed in order to inspect and document any medial or lateral pedicle breaches that occurred and the integrity of the surrounding neurovascular structures for each respective screw. A pedicle breach was defined a priori as any disruption of the cortical wall (medial, lateral, superior, or inferior) of the pedicle by a screw. Although documented during open dissection, breach of the lateral vertebral body (VB) wall for CS placement was not considered or combined with pedicle breaches in this study as some have considered this
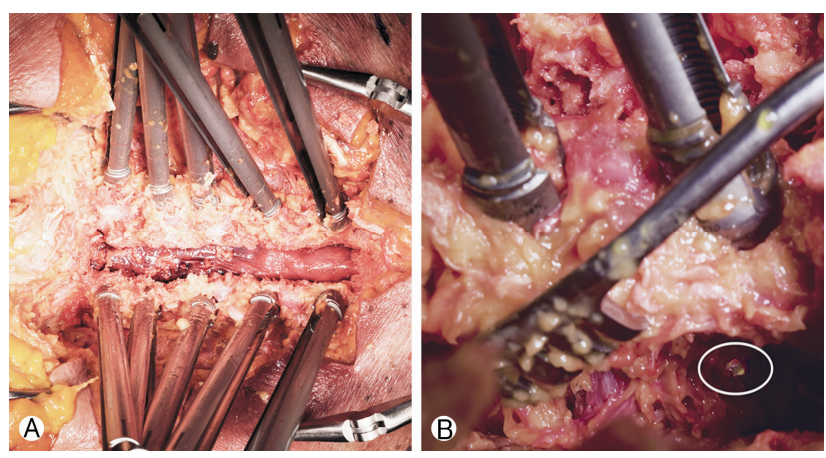

Fig. 2. Anatomic open-dissection of the pedicle screw (PS, top of panel (A) and cortical screw (CS, bottom of panel (A) constructs for breach incidence determination after posterior midline approach and laminectomy. Although no pedicle breaches were observed, one CS was found to have breached the posterior third of lateral vertebral body (highlighted in panel (B)). No foraminal or extra-spinal neurovascular structures were seen to have been compromised as a result. 
ideal due to the added cortical bone purchase. Note of this was made, however, to examine for potential extra-spinal neurovascular compromise. One MIS-trained spine surgeon (senior author, J.R.) was responsible for all PS and CS placement in order to decrease inter-observer (surgeon-specific) variability. A separate surgeon (A.J.) was responsible for open dissection and recording screw breach in order to decrease confirmation bias (surgeon-based bias from having placed the screws). No blinding for the open dissection was possible as immediate recognition of which screw was a PS or CS was possible based on anatomical landmarking of entry points.

\section{RESULTS}

Upon open dissection, no pedicle breaches of either construct were found. As such no statistical comparison was possible. A post-hoc power analysis completed (G*Power software) ${ }^{4)}$ revealed a required total sample size of 760 instrumented levels (380 levels of both PS and CS for comparison) based on a calculated power of 0.8 , significance of 0.05 , and $6 \%$ incidence of PS breach versus $12 \%$ incidence of CS breach. The latter incidence of PS breach was used based on a previous, relatively large study, examining the incidence of fluoroscopically-guided MIS-PS placement breach ${ }^{20)}$. The incidence of CS breach was arbitrarily chosen for the power calculation as being double that of PS. That being said however, numerous previous studies have not found a difference in breach incidence, nor mechanical failure of PS versus CS placement ${ }^{2,3,13,17)}$.

At one level, a CS could be seen protruding though the lateral VB wall (highlighted in Fig. 2B). The lateral VB breach by the CS occurred in the posterior-third of the VB just under the superior end-plate. Two exposed screw threads could be visualized which were well away from entering/exiting foraminal structures or any paraspinal neurovascular structures or entering/exiting foraminal structures.

\section{DISCUSSION}

Spinal instrumentation for stabilization has greatly evolved over the past few decades. Since their original description by Roy-Camille et al. traditional PS have been considered the gold standard for posterior spinal stabilization ${ }^{18)}$. PS placement, however, does have inherent risk and in the case of open placement, necessitates lateral paraspinal tissue dissection. More recently, a new cortical bone trajectory method of pedicle screw placement has emerged ${ }^{13,14,17,19)}$ with reported benefits including: more cortical bone purchase resulting in superior biomechanical strength ${ }^{1,17,21)}$, equivalent fusion rate ${ }^{13)}$, and decreased surgical morbidity ${ }^{13)}$. In the same vein, a trend toward decreasing operative morbidity through more minimally invasive techniques has also emerged ${ }^{5,6,8,11,12,16,20)}$. The combination of the latter two concepts, more specifically posterior spinal stabilization through percutaneous cortical screws, has not been previously described with subsequent anatomical investigation for complications of the CS construct. Here, we detail this novel technique, its advan- tages and disadvantages, and compare its incidence of pedicle breach with that of percutaneous PS.

Although one previous study suggested that a theoretically higher incidence of pedicle breach may be observed with CS, this was not found in our study ${ }^{3,7)}$. In another study completed by Santoni et al. a relatively high incidence of pedicle breach was found, however CS were inserted using a free-hand technique without the use of fluoroscopy which is required for percutaneous instrumentation ${ }^{19)}$. As such, their reported incidence of pedicle breach is more likely related to the lack of imaging and less likely the anterolateral trajectory ${ }^{19}$. With image guidance, the incidence of percutaneous PS breach has been found to be approximately $6 \%{ }^{20)}$. Although the incidence of percutaneous CS breach is unknown (making an appropriate sample size difficult to estimate), this technical paper is under-powered (as shown through the included post-hoc analysis). It is possible that the increasing use of CS could lead this complication becoming more commonplace and result in it falling out of favor. Another explanation for the lack of difference between groups seen could be related to the study design. In order to decrease technique variability, all screws were placed by one senior surgeon whom specializes in MIS spine surgery. This may have resulted in a lower than expected pedicle breach incidence and decreased the generalizability of our findings. However, the aim of this study was primarily to determine the feasibility and describe the technique itself, rather than provide a comprehensive study comparing PS and CS. Further adequately powered study investigating the different complication profiles of each technique would be required to establish such conclusions more definitively. Other disadvantages to the CS technique relate to the anatomical characteristics of its trajectory. Adopting a CBT inherently includes potential lateral VB breach complications (although this has also been said to be advantageous as it increases screw purchase by including another cortical bone surface). In the one example of this in our paper, however, the breach occurred away from any spinal or paraspinal neurovascular structures. Finally, and perhaps most importantly, use of CS results in a medial screw-head which can interfere with neural decompression and potentially require screw placement after decompression (which has its own respective drawbacks). Moreover, bony decompression may result in weakening of the CS construct and predispose to failure or pars fracture. This problem would be mitigated, however, with CS placement after lateral interbody fusion and indirect decompression.

The lateral-to-medial trajectory of PS makes it difficult to place in a lateral position. However, the medial-to-lateral trajectory of CS makes it ideal for such a case. Placement of CS would then facilitate screw insertion after lateral interbody fusion without having to reposition the patient prone. In a randomized controlled trial comparing PS and CS, Lee et al. demonstrated that CS also result in less surgical morbidity, blood loss, and shorter length of stay potentially due to less lateral dissection being required ${ }^{13)}$. These benefits would be especially pronounced in morbidly obese patients in whom our described approach could be particularly well-suited for. However, the study by Lee 
et al. consisted of an open approach which is an important difference compared to our percutaneous CS technique. Moreover, in their study Lee et al. also found a decreased incidence of facet capsule violation with the use of CS versus PS. Here, although we did not observe any differences between the two constructs upon open inspection with respect to facet violation, it is possible that the more inferomedial entry point with percutaneous CS could result in this occurrence as well. To avoid this, it would be important to ensure perfect AP radiographs are obtained prior to instrumentation, as well as and selecting a starting point akin to that of the open CBT with more acute screw trajectory angulation. In a similar, albeit different study, Orita et al. describe a percutaneous method for placing $\mathrm{CS}^{16}$. This was done as a prospective cohort study comparing percutaneous pedicle screw fixation (PPS) to percutaneous cortical screw fixation (PCS) with "mini-open" TLIF in patients undergoing single-level fusion for spondylolisthesis or lateral disc herniation. The authors state that there were no complications (aggregate of all potential patient and implant related complications) in either group. In comparison, our study is an anatomical study allowing us the unique advantage of performing an open dissection after instrumentation to compare instrumentation-related complications via the gold-standard, direct visualization. Our study supports and compliments this previous clinical study in that it provides a basic, anatomical foundation to support their findings and this novel instrumentation technique. Furthermore, in their study Orita et al. also make note of the difficulty that can be encountered with achieving an acute medial to lateral angle for PCS due to the intact midline structures. Here, we adopted a less acute of lateral and rostral angulation (akin to a hybrid of a traditional CBT and "bulls-eye" technique as previously described) ${ }^{16,20)}$ and contend that this trajectory obviates any difficulty in achieving the desired trajectory, as well as allows for larger screws to be placed which would increase construct strength.

Several biomechanical studies have been completed examining pullout and toggle characteristics of CS and PS with CS having increased insertional torque, pullout strength, and equivalent construct rigidity as PS-based constructs ${ }^{10,14,15,19)}$. These biomechanical properties have led to CS being investigated as a rescue option in the event of PS failure. In their study, Calvert et al. demonstrated through cadaveric biomechanical testing that CS placed to rescue failed PS retained similar pullout strength and stiffness as the original PS. Studies such as this further expand the potential application and advantages of CS placement to include revision surgery in high-risk patients such as osteopenic or osteoporotic patients, in whom $\mathrm{CS}$ techniques are already said to be advantageous ${ }^{2,19)}$.

\section{CONCLUSION}

The advancement of MIS spine techniques and the advent of instrumentation methods such as CS-based constructs continue to expanded patient care. Here, we report a novel study of percutaneous CS insertion with open cadaveric dissection comparing it to PS, incorporating the advantages of both MIS and CS fixation. Although feasible and technically comparable to PS in our study, further investigation comparing these two techniques and rigorous patient selection for its application are necessary.

Conflict of Interest: The authors have nothing to disclose, nor conflicts of interest related to this article, and the contents of this manuscript have not been previously published.

\section{REFERENCES}

1. Amaritsakul Y, Chao CK, Lin J: Comparison study of the pullout strength of conventional spinal pedicle screws and a novel design in full and backed-out insertions using mechanical tests. Proc Inst Mech Eng H 228:250-257, 2014

2. Calvert GC, Lawrence BD, Abtahi AM, Bachus KN, Brodke DS: Cortical screws used to rescue failed lumbar pedicle screw construct: a biomechanical analysis. J Neurosurg Spine 22:166172, 2015

3. Chin KR, Pencle FJR, Coombs AV, Elsharkawy M, Packer CF, Hothem EA, et al: Clinical outcomes with midline cortical bone trajectory pedicle screws versus traditional pedicle screws in moving lumbar fusions from hospitals to outpatient surgery centers. Clin Spine Surg 30:E791-E797, 2017

4. Faul F, Erdfelder E, Lang AG, Buchner A: G*Power 3: a flexible statistical power analysis program for the social, behavioral, and biomedical sciences. Behav Res Methods 39:175-191, 2007

5. Fessler RG, Khoo LT: Minimally invasive cervical microendoscopic foraminotomy: an initial clinical experience. Neurosurgery 51:S37-45, 2002

6. Fessler RG, O'Toole JE, Eichholz KM, Perez-Cruet MJ: The development of minimally invasive spine surgery. Neurosurg Clin N Am 17:401-409, 2006

7. Glennie RA, Dea N, Kwon BK, Street JT: Early clinical results with cortically based pedicle screw trajectory for fusion of the degenerative lumbar spine. J Clin Neurosci 22:972-975, 2015

8. Goldstein CL, Macwan K, Sundararajan K, Rampersaud YR: Perioperative outcomes and adverse events of minimally invasive versus open posterior lumbar fusion: meta-analysis and systematic review. J Neurosurg Spine 24:416-427, 2016

9. Goldstein MJ, Bruffey J, Eastlack RK: New minimally invasive technique for direct pars interarticularis osteosynthesis using cortical screws and spinous-process modular link. Spine (Phila Pa 1976) 41:E1421-E1424, 2016

10. Inceoglu S, Montgomery WH, Jr., St Clair S, McLain RF: Pedicle screw insertion angle and pullout strength: comparison of 2 proposed strategies. J Neurosurg Spine 14:670-676, 2011

11. Isaacs RE, Podichetty VK, Santiago P, Sandhu FA, Spears J, Kelly $\mathrm{K}$, et al: Minimally invasive microendoscopy-assisted transforaminal lumbar interbody fusion with instrumentation. J Neurosurg Spine 3:98-105, 2005

12. Khoo LT, Palmer S, Laich DT, Fessler RG: Minimally invasive percutaneous posterior lumbar interbody fusion. Neurosurgery 51:S166-181, 2002

13. Lee GW, Son JH, Ahn MW, Kim HJ, Yeom JS: The comparison of pedicle screw and cortical screw in posterior lumbar interbody 
fusion: a prospective randomized noninferiority trial. Spine J 15:1519-1526, 2015

14. Matsukawa K, Yato Y, Kato T, Imabayashi H, Asazuma T, Nemoto K: In vivo analysis of insertional torque during pedicle screwing using cortical bone trajectory technique. Spine (Phila Pa 1976) 39:E240-245, 2014

15. Mobbs RJ: The "medio-latero-superior trajectory technique": an alternative cortical trajectory for pedicle fixation. Orthop Surg 5:56-59, 2013

16. Orita S, Inage K, Kubota G, Sainoh T, Sato J, Fujimoto K, et al: One-year prospective evaluation of the technique of percutaneous cortical bone trajectory spondylodesis in comparison with percutaneous pedicle screw fixation: A preliminary report with technical note. J Neurol Surg A Cent Eur Neurosurg 77:531537, 2016

17. Perez-Orribo L, Kalb S, Reyes PM, Chang SW, Crawford NR: Biomechanics of lumbar cortical screw-rod fixation versus pedi- cle screw-rod fixation with and without interbody support. Spine (Phila Pa 1976) 38:635-641, 2013

18. Roy-Camille R, Saillant G, Mazel C: Internal fixation of the lumbar spine with pedicle screw plating. Clin Orthop Relat Res: 7-17, 1986

19. Santoni BG, Hynes RA, McGilvray KC, Rodriguez-Canessa G, Lyons AS, Henson MA, et al: Cortical bone trajectory for lumbar pedicle screws. Spine J 9:366-373, 2009

20. Smith ZA, Sugimoto K, Lawton CD, Fessler RG: Incidence of lumbar spine pedicle breach after percutaneous screw fixation: a radiographic evaluation of 601 screws in 151 patients. J Spinal Disord Tech 27:358-363, 2014

21. Wray S, Mimran R, Vadapalli S, Shetye SS, McGilvray KC, Puttlitz CM: Pedicle screw placement in the lumbar spine: effect of trajectory and screw design on acute biomechanical purchase. J Neurosurg Spine 22:503-510, 2015 